\title{
Validation par les experts et évaluation de l'efficacité d'un jeu éducatif en ligne sur les attitudes des jeunes de 13 et 14 ans à l'égard de la santé sexuelle
}

On-line educational game : validation by the experts and evaluation of the impact of the game on the attitudes of the young people towards the sexual health Validación por expertos y evaluación de la eficiencia de un juego educativo en línea sobre las actitudes de los jóvenes de 13 y 14 años respecto a la salud sexual

\section{Lise Renaud et Louise Sauvé}

Volume 36, numéro 3, 2010

Éducation à la santé

Texte reçu le : 7 avril 2009, version finale reçue le : 6 juin 2010, accepté le : 4 août 2010

URI : https://id.erudit.org/iderudit/1006251ar

DOI : https://doi.org/10.7202/1006251ar

Aller au sommaire du numéro

Éditeur(s)

Revue des sciences de l'éducation

ISSN

0318-479X (imprimé)

1705-0065 (numérique)

Découvrir la revue

Citer cet article

Renaud, L. \& Sauvé, L. (2010). Validation par les experts et évaluation de l'efficacité d'un jeu éducatif en ligne sur les attitudes des jeunes de 13 et 14 ans à l'égard de la santé sexuelle. Revue des sciences de l'éducation, 36(3), 671-694. https://doi.org/10.7202/1006251ar

\section{Résumé de l'article}

Cet article vise à justifier la pertinence d'un jeu éducatif en ligne intervenant sur les attitudes en matière de santé sexuelle. La recherche de processus auprès de dix experts permet d'examiner, entre autres, la justesse du contenu et l'adéquation du jeu au public cible ; quant à la recherche d'efficacité, elle vise à cerner, au moyen d'un questionnaire (pré-post test) contenant 34 indicateurs, les attitudes de 173 jeunes de 13 à 14 ans envers la santé sexuelle. La recherche de processus révèle l'adéquation entre le jeu et les réalités des jeunes. Les résultats d'efficacité indiquent une amélioration des attitudes à l'égard de la santé sexuelle. 


\title{
Validation par les experts et évaluation de l'efficacité d'un jeu éducatif en ligne sur les attitudes des jeunes de 13 et 14 ans à l'égard de la santé sexuelle*
}

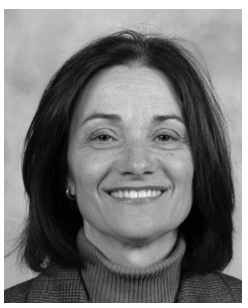

Lise Renaud, professeure Université du Québec à Montréal



Louise Sauvé, professeure TÉLUQ, Université du Québec à Montréal

\begin{abstract}
RÉSUMÉ • Cet article vise à justifier la pertinence d'un jeu éducatif en ligne intervenant sur les attitudes en matière de santé sexuelle. La recherche de processus auprès de dix experts permet d'examiner, entre autres, la justesse du contenu et l'adéquation du jeu au public cible; quant à la recherche d'efficacité, elle vise à cerner, au moyen d'un questionnaire (pré-post test) contenant 34 indicateurs, les attitudes de 173 jeunes de 13 à 14 ans envers la santé sexuelle. La recherche de processus révèle l'adéquation entre le jeu et les réalités des jeunes. Les résultats d'efficacité indiquent une amélioration des attitudes à l'égard de la santé sexuelle.
\end{abstract}

MOTS CLÉS • jeu éducatif en ligne, santé sexuelle, éducation à la santé, attitudes, jeunes.

\section{Introduction et problématique}

Le Centre d'expertise et de recherche sur l'apprentissage à vie (SAVIE) a développé des environnements de conception de jeux éducatifs en ligne (Web), dont l'objectif est d'intervenir sur les connaissances, les attitudes et les comportements en matière de santé. Cet article relate en particulier la démarche de validation d'un jeu portant sur les infections transmissibles sexuellement (ITS) ainsi que

* Cette recherche a été subventionnée par le Conseil de recherche en sciences sociales et humaines (CRSH, 2003-2008). Nous désirons remercier le Dr Fernand Cantin et le Dr Martin Delage, omnipraticiens, pour avoir réalisé la première version du jeu, sans lequel les expérimentations n’auraient pu avoir lieu. De plus, nous remercions les écoles qui ont bien voulu nous accueillir et nous permettre de réaliser l'expérimentation du jeu auprès de leurs élèves. Enfin, nous remercions Gabriela Hanca, Jean-Philippe Laperrière et Jérôme Elissalde qui ont réalisé la collecte des données. 
l'évaluation de son efficacité sur le plan des attitudes auprès d'élèves du secondaire.

Alors que la recension des écrits discute beaucoup du potentiel créatif des nouvelles technologies, dont les jeux éducatifs en ligne, le constat qui est fait, côté apprenant, est celui de la mise en veilleuse des nouvelles aptitudes susceptibles d'être développées. Selon Prensky $(2001,2005)$, ces aptitudes sont présentes dans la génération née à partir de 1980. Cette génération a développé une manière d'apprendre et de structurer l'information différente des générations précédentes. Les jeunes apprendraient plus facilement en expérimentant, seraient compétents à accomplir plus d'une tâche à la fois et ils dépendraient des technologies pour accéder à l'information ou pour socialiser. Ce constat a été également réitéré par Oblinger et Oblinger (2005) et le CÉFRIO (2009).

Il semble que l'école n'explore pas le potentiel éducatif de ces nouveaux usages qui s'inspirent d'une approche constructiviste. Bien qu'il existe du matériel pédagogique dans les écoles qui soutiennent cette approche, la plupart n'offrent pas de contenus multimédias et interactifs (Gauthier, Mellouki, Simard, Bissonnette et Richard, 2004) et encore moins des jeux en ligne. Dans ce contexte, une recherche Apprendre par les jeux du réseau SAGE (Simulations and Advanced Gaming Environments for learning project / ApprentisSAGE par les jeux et simulations) avait pour but de développer et d'expérimenter dans les écoles des jeux en ligne en matière de prévention et de promotion de la santé, afin d'identifier et de documenter ce qui favorise ou inhibe le changement d'attitudes.

L'article porte à la fois sur la validation par les experts d'un jeu créé à l'aide d'un environnement de conception de jeux éducatifs en ligne et sur l'évaluation de l'efficacité de ce jeu, conçu par deux médecins, au sujet de la prévention de la maladie et de la promotion de la santé en regard des infections transmissibles sexuellement, en termes de changements d'attitudes auprès des élèves du secondaire âgés de 13 à 14 ans.

\subsection{Situation des infections transmissibles sexuellement et par le sang chez les jeunes Québécois}

L'expression infection transmissible sexuellement et par le sang a aujourd'hui supplanté dans l'usage celle de maladie transmise sexuellement, car elle est plus inclusive (elle comprend les infections asymptomatiques et certaines infections qui se transmettent par le sang). Par infection transmissible sexuellement et par le sang, nous entendons notamment la chlamydia, l'infection gonococcique, la lymphogranulomatose vénérienne, la syphilis infectieuse, l'hépatite B, l'hépatite C et l'infection par le VIH (virus de l'immunodéficience humaine).

$\mathrm{Au}$ Québec, après avoir observé des diminutions importantes du nombre d'infections transmissibles sexuellement et par le sang, au début des années 1990, on assiste, depuis 1998, à une augmentation de plus de $70 \%$ des taux de chlamydia et de gonorrhée chez les jeunes Québécois âgés de 15 à 24 ans. En 2008, quelque 
9462 cas déclarés de chlamydia et 754 cas de gonorrhée (Gouvernement du Québec, 2008). Il s'agit là des données les plus récentes.

Les jeunes adultes constituent un des groupes les plus vulnérables à l'une ou l'autre des infections transmissibles sexuellement et par le sang. En effet, en 2007, les jeunes âgés entre 15 et 24 ans représentent $65 \%$ des cas de chlamydiose génitale déclarés, $41 \%$ des cas d'infection gonococcique, $9 \%$ des cas d'hépatite $B, 7 \%$ des cas de syphilis infectieuse et $6 \%$ des cas d'hépatite $C$. Par ailleurs, ils totalisent $5 \%$ de l'ensemble des nouveaux et anciens cas reconnus. De plus, cinq cas de syphilis infectieuse ont été signalés chez des adolescents appartenant au groupe des 15-19 ans en 2007, et on estimait à l'époque que sept le seraient en 2008 (Gouvernement du Québec, 2008).

Selon une étude canadienne (Conseil des ministres de l'Éducation du Canada, 2003 : voir Lacroix et Cloutier, 2006), en 2002, $23 \%$ des garçons et $19 \%$ des filles de troisième secondaire (âge moyen 14 ans) affirment avoir eu des rapports sexuels au moins une fois dans leur vie. Selon une enquête de 2005-2006 (Gouvernement du Québec, 2008), $72 \%$ des cégépiens montréalais (âge moyen 18 ans) ont eu, au moins une fois dans leur vie, une relation sexuelle orale, vaginale ou anale; l'âge moyen pour la première relation sexuelle vaginale est 16 ans.

Le Conseil des ministres de l'Éducation du Canada (2003; Lacroix et Cloutier, 2006) a publié une étude sur la santé sexuelle des jeunes, qui montre que l'insuffisance des connaissances à propos des infections transmissibles sexuellement et par le sang semble être l'aspect le plus problématique. En effet, la majorité des élèves de troisième à cinquième secondaire croient qu'il est impossible de contracter, une autre fois, la même infection transmissible sexuellement et par le sang, tandis qu'environ la moitié d'entre eux ne savent pas que celles-ci peuvent être asymptomatiques. De plus, moins de la moitié ignorent que la Vaseline ${ }^{\mathrm{mc}}$ n'est $^{\prime}$ pas un lubrifiant recommandé avec le condom. En termes d'attitudes, il semble que la crainte des répercussions négatives des infections transmissibles sexuellement et par le sang sur la santé n'ait que peu à voir avec leur décision d'être sexuellement actifs. Selon une enquête, les jeunes de 15 à 18 ans (Turenne, 2004; Lacroix et Cloutier, 2006) sont très conscients du VIH (transmission par le sang, d'où le dernier $S$ de ITSS) et de sa protection, mais beaucoup moins préoccupés par les autres infections transmissibles sexuellement et renseignés sur ces dernières. D'où notre intérêt de nous attarder, auprès des jeunes du secondaire, non pas à toutes les infections transmissibles sexuellement et par le sang, mais seulement aux infections transmissibles sexuellement.

\subsection{Les stratégies de promotion de la santé et de prévention de la maladie}

Étant donné la recrudescence des infections transmissibles sexuellement et par le sang au Québec et leurs répercussions considérables, en particulier chez les jeunes, il est essentiel de continuer à offrir des interventions en matière de prévention de la maladie et de promotion de la santé. Le milieu scolaire, le milieu 
communautaire et les cliniques médicales sont des lieux que les jeunes fréquentent et où les intervenants, les enseignants et les praticiens peuvent encadrer et guider les jeunes, dans le respect de leur individualité.

Les stratégies de prévention de la maladie et de promotion de la santé peuvent notamment se déployer par l'éducation à la santé, l'organisation communautaire et les communications pour la santé. L'éducation à la santé est un terme employé pour désigner les occasions d'apprentissage délibérément mises en œuvre pour faciliter les changements d'attitudes et de comportements, en vue d'atteindre un objectif déterminé à l'avance. Ce processus vise à motiver les gens à se préoccuper de la santé dans un sens personnel et dans une perspective écologique (Renaud et Sauvé, 1990). L'éducation à la santé repose avant tout sur les relations interpersonnelles: l'information s'adresse à un auditoire précis et vise à augmenter les connaissances de celui-ci, à modifier ses attitudes, à développer ses habiletés; bref, à favoriser une décision responsable et une prise de conscience de ce qui est bon pour soi (Caron-Bouchard et Renaud, 2001). D'une part, il s'agit d'offrir aux jeunes des moyens concrets de se prendre en main et, d'autre part, de fournir des moyens aux accompagnateurs de ces jeunes pour faire cheminer ces derniers. Plusieurs moyens peuvent être utilisés: un exposé en classe, une discussion en groupe, une formation de groupe de pairs, un jeu éducatif ou encore un jeu numérique interactif. Renaud et Sauvé (1990) montrent que les moyens éducatifs novateurs, comme les jeux et les simulations, sont mieux adaptés pour changer les attitudes que les techniques plus classiques. En effet, ces moyens permettent un transfert de pouvoir à la personne en ce sens qu'ils lui donnent le sentiment d'avoir du pouvoir sur elle-même et sur sa vie. Garris, Ahlers et Driskell (2002, p. 457) décrivent les apprentissages de type affectif développés par le jeu: le sentiment de confiance en soi, les attitudes, les préférences et les dispositions. Hornung et collab. (2000) ont expérimenté diverses activités sur cédérom (vidéo, dessins animés) pour amener les élèves à adopter des attitudes préventives envers le cancer de la peau. Les résultats montrent que ces activités ont effectivement développé des attitudes préventives.

\subsection{L'efficacité des jeux numériques comme outils en éducation à la santé}

Selon plusieurs études récentes, les jeux éducatifs numériques (à partir d’un logiciel ou en ligne) conduisent à un changement d'attitudes.

Selon Bijker, Van Buuren et Wynants (2006), les attitudes reflètent les influences du milieu social sur la personne et se traduisent par des évaluations automatiques de situations. Bottino, Ferlino, Ott et Tavella (2007) notent un changement d'attitudes chez les joueurs qui ont participé aux jeux (PappaLOTTO, Hexip, Studio 5 et Magic Bass) par rapport au groupe témoin: la participation aux jeux a affecté l'attitude globale envers les différents thèmes de santé. Tingstrom, Sterling-Turner et Wilczynski (2006) ont réalisé une recension des écrits portant sur l'utilisation du jeu Good Behavior Game sur une période allant de 1969 à 2002. 
Ils affirment que les participants ont adopté notamment des attitudes adéquates qui faisaient l'objet d'apprentissage dans le jeu. Ainsi, l'intégration de contenus d'apprentissage dans les jeux peut entraîner des changements d'attitudes.

La méta-analyse de Bailey, Murray, Rait, Mercer, Morris, Peacock, Cassell et Nazareth (2009) sur l'efficacité de 15 interventions en ligne au sujet de la promotion de la santé sexuelle montre un effet majeur sur les connaissances et un léger effet sur les attitudes des participants, comparativement à une intervention en face à face. Cette méta-analyse relate les effets positifs des interventions sur les attitudes à l'égard de la santé sexuelle:

- perception de sa propre vulnérabilité à l'égard des ITS (Jenkins, 2000; Bailey, Murray, Rait, Mercer, Morris, Peacock, Cassell et Nazareth, 2009);

- amélioration dans ses communications interpersonnelles (Kann, 1987; Bailey et collab., 2009);

- attitude plus favorable dans le délai des relations sexuelles (Van Laar, 2000; Bailey et collab., 2009);

- augmentation des bénéfices de la négociation d'une sexualité sécuritaire (Davidovich, 2006; Bailey et collab., 2009);

- accroissement des bénéfices physiques et sociaux (Evans, 2000; Bailey et collab., 2009);

- perception accrue des effets sécuritaires de l'utilisation du condom (Bowen, Horvath et Williams, 2007; Bailey et collab., 2009).

D'après les résultats de cette méta-analyse, plusieurs interventions n'ont pas généré d'effets sur les attitudes ou, au contraire, ont provoqué des effets négatifs:

- aucune augmentation dans sa capacité de parler du condom (Kann, 1987; Bailey et collab., 2009;

- aucune nouvelle attitude ni norme sociale modifiée en regard de l'usage du condom (Keine et Barta, 2006; Mikolajczak, 2008; Bailey et collab., 2009);

- aucune différence sur l'ensemble des attitudes à l'égard des infections transmissibles sexuellement (Read, Miller, Appleby, Nwosu, Reynaldo et Lauren, 2006; Bailey et collab., 2009);

- aucun effet sur une échelle d'attitudes à propos des risques sexuels (Van Laar, 2000; Bailey et collab., 2009);

- négation de la possibilité d'avoir une infection transmissible sexuellement (Roberto, Zimmerman, Carlyle, Abner, Cupp et Hansen, 2007; Bailey et collab., 2009).

Cette inconsistance dans les résultats peut être due à l'instrument servant à mesurer les attitudes; par exemple, selon la méta-analyse citée:

- existence de plusieurs échelles d'attitudes à l'égard de la sexualité (Alemi, Cherry et Meffert, 1989; Bailey et collab., 2009); 
- difficulté de mesurer le confort dans une communication interpersonnelle franche (Kann, 1987; Bailey et collab., 2009);

- mesure liée à la sécurité affective à la suite d'un échange verbal (Davidovich, de Wit et Stroebe, 2006; Bailey et collab., 2009);

- diversité des mesures sur les attitudes envers les préservatifs (Bowen et collab., 2007; Van Laar, 2000; Bailey et collab., 2009);

- échelles d'approbation du port du condom par les pairs (Jenkins et collab., 2000; voir Bailey, Murray et collab., 2009), par la famille et les amis (Keine et Barta, 2006; Bailey et collab., 2009);

- mesures des attitudes à l'égard d'un examen annuel de sa santé sexuelle (Mikolajczak, Kok et Hospers, 2008; Bailey et collab., 2009);

- attitude envers l'abstinence jusqu'au mariage (Roberto et collab., 2007; Bailey et collab., 2009);

- sensibilité à l'idée d'avoir une grossesse, une infection transmissible sexuellement ou le VIH (Jenkins et collab., 2000; Roberto et collab., 2007; Bailey et collab., 2009).

Par ailleurs, certains auteurs se montrent prudents sur l'efficacité de ce moyen sur l'apprentissage ou le changement d'attitudes en santé (Rassin, Gutman et Silner, 2004; Barab et collab., 2005; Baldaro, Tuozzi, Codispoti et Montebarocci, 2004).

Tenant compte de ces résultats, il s'avère fondamental de procéder à des études qui permettent de mesurer de façon rigoureuse l'efficacité du jeu pour les attitudes. Ainsi, l'étude que nous présentons avait pour but d'évaluer l'efficacité d'un jeu, conçu par des enseignants et des praticiens de la santé pour la prévention et la promotion de la santé, en termes de changements d'attitudes auprès d'élèves de 13 à 14 ans. Dans le présent article, nous tenterons de répondre à la question suivante: Quel type d'apprentissage lié aux attitudes les jeux éducatifs en ligne favorisent-ils en santé?

Nous présenterons le cadre théorique en précisant ce qu'est un jeu éducatif et en quoi les jeux éducatifs peuvent influencer les attitudes en matière de santé. Notre question de recherche suivra. Puis nous exposerons notre méthodologie de type pré-post test groupe simple auprès de 173 jeunes âgés de 13-14 ans. Ensuite, nous détaillerons nos résultats de recherche, et enfin nous discuterons de la pertinence d'un jeu interactif en ligne pour modifier les attitudes des jeunes à l'égard de la santé sexuelle.

\section{Contexte théorique}

Afin de répondre à notre question générale de recherche, nous avons examiné les éléments constitutifs d'un jeu éducatif en santé, ainsi que les théories qui traitent du changement d'attitudes en santé. 


\subsection{Définition du jeu éducatif en ligne}

Notre recension des écrits scientifiques au sujet de l'efficacité des jeux éducatifs sur la santé s'est appuyée sur une définition du jeu éducatif: une situation artificielle (fictive, fantaisiste) qui, tout en possédant le potentiel de favoriser des apprentissages, place un ou plusieurs joueurs en position de conflit (lutte, confrontation) soit les uns par rapport aux autres, soit tous ensemble (coopération) contre d'autres forces. Ces joueurs doivent respecter les règles (procédure, contrôle et clôture) qui structurent leurs actions en vue d'un but déterminé: soit gagner (gagnant contre perdant), être victorieux (contre le hasard, l'ordinateur, un ou plusieurs joueurs), soit prendre leur revanche contre un adversaire (Sauvé, Renaud, Kaufman, Samson, Bluteau-Doré, Dumais, Bujold, Kaszap et IsaBelle, 2005).

Dans cette étude, nous nous intéressons particulièrement aux jeux éducatifs qui ont été créés à l'aide des langages de programmation Web. Ces jeux n'exigent aucun téléchargement et sont accessibles uniquement par Internet.

\subsection{Développement d'un jeu en ligne intervenant sur les attitudes}

Lieberman (1997) souligne l'importance de développer des jeux sur le thème de la santé, qui soient fondés sur les théories sociales cognitives, et de respecter les principes de la promotion de la santé, où l'individu est mis en position de faire pour lui-même les choix les plus éclairés. En matière de santé, les changements d'attitudes sont des objectifs importants, car les attitudes favorisent un apprentissage affectif. Cela s'avère d'autant plus essentiel pour des problématiques liées à la sexualité, qui ont un caractère intime, si bien qu'admettre ouvertement une activité sexuelle ou une orientation sexuelle peut être difficile en raison du stigmate ou du secret. Ainsi, les attitudes méritent d'être considérées parce qu'elles interviennent dans la formation du contexte dans lequel le comportement sexuel arrive.

Nous devons distinguer ici entre attitude et comportement. Une attitude est une orientation générale de la manière d'être d'un individu face à certains éléments $d u$ monde (Mucchielli, 2002, p. 19). Par exemple, on peut avoir une attitude méfiante envers une personne plus âgée que soi, mais adopter une attitude de confiance face à un jeune de son âge. C'est une disposition mentale explicative du comportement, mais ce n'est pas le comportement (Mucchielli, 2002; Renaud et Sauvé, 1990). Les attitudes comportent trois composantes majeures: une composante cognitive, qui concerne les croyances, ou encore les préjugés d'un individu, à propos d'un objet ou d'un sujet; une composante affective, qui renvoie au sentiment de rejet ou d'acceptation à l'égard d'un objet ou d'un sujet; et une composante comportementale, qui se définit comme la prédisposition d'un individu à agir quand l'objet ou le sujet de l'attitude lui est présenté (Caron-Bouchard et Renaud, 2001). Par exemple, croire que l'on est vulnérable à une infection transmissible sexuellement; croire qu'on sera mal vu si on propose le condom; percevoir qu'une infection transmise sexuellement est une menace pour soi. Quant 
au comportement, il se définit comme un ensemble de réactions d'un individu qui sont observables objectivement (Le Petit Larousse illustré, 2004): par exemple, l'individu achète un condom.

D'autres articles de recherche portent sur les effets des connaissances de ce jeu sur les jeunes. Pour notre part, nous avons voulu examiner en profondeur l'effet du jeu sur les attitudes, car très peu d'articles dans les écrits de recherche s'intéressent à cette question fort pertinente.

Différentes théories nous permettent de dégager les composantes à intégrer à un jeu éducatif afin que celui-ci puisse influencer les attitudes. D’après la théorie du renforcement, la théorie de la dissonance ainsi que la théorie de l'inoculation (Petty, Cacioppo et Heesacker, 1981; Renaud et Sauvé, 1990), les activités d'apprentissage doivent permettre à l'apprenant de jouer 1) un rôle réel ou fictif qui lui permet 2) de se confronter à des positions similaires ou différentes de la sienne 3) en interaction avec ses pairs tout en ayant 4) une rétroaction qui lui permette de se réajuster au fur et à mesure de son apprentissage. Ces quatre composantes se retrouvent en grande partie dans le jeu de rôle, considéré comme une activité qui a de l'influence sur le changement d'attitudes (Chauvin, 2001; Renaud et Sauvé, 1990).

Le jeu de rôle est une technique pédagogique issue de la méthode active et permettant à la personne de faire des découvertes en se mettant elle-même en scène, en jouant un rôle fictif ou réel (Chauvin, 2001). Dans notre étude, nous reprenons la définition de Chauvin (2001), à savoir que le jeu de rôle est un échange verbal ou écrit, au cours duquel un ou plusieurs individus comprennent comment et pourquoi ils agissent dans une situation donnée, réelle ou imaginaire, présente, passée ou future, par un jeu spontané sur un canevas. Ainsi, les activités du jeu que nous avons expérimentées visaient à offrir une grande marge de manœuvre à l'apprenant, à favoriser tantôt l'improvisation, tantôt la défense d'une position similaire ou différente de la sienne, à mettre en place les conditions qui permettent une interaction entre les joueurs, à fournir à l'individu des pistes argumentaires et à lui offrir une rétroaction afin qu'il puisse réajuster, s'il y a lieu, sa position.

Compte tenu que le jeu éducatif faisant l'objet de notre expérimentation vise à changer les attitudes, il devra donc offrir des activités fondées sur les paramètres d'un jeu de rôle. En d'autres mots, les activités du jeu consistent, pour l'apprenant, à jouer un rôle plus ou moins structuré selon le type de changement d'attitudes souhaité. Il est appelé à défendre une position similaire à la sienne ou différente de la sienne. Certaines activités lui permettent de jouer ce rôle en équipe ou en compétition avec un autre joueur. Une fois l'improvisation achevée, les autres joueurs, à l'aide d'un corrigé, peuvent commenter la performance (rétroaction) de l'apprenant et lui accorder ou non des points.

Compte tenu des éléments d'apprentissage identifiés pour modifier les attitudes à l'aide des jeux, cette étude vise de façon plus spécifique à examiner dans quelle mesure les quatre éléments clés du jeu - improvisation, argumentation, confron- 
tation dans l'interaction et rétroaction - réussissent à changer les attitudes des jeunes de 13 et 14 ans.

\subsection{Question de recherche et hypothèse}

La question spécifique est: Le jeu ITS: Stopper la transmission favorise-t-il un changement des attitudes envers les infections transmissibles sexuellement chez les jeunes? Notre hypothèse est qu'il y aura une différence observable dans l'attitude des jeunes envers les infections transmissibles sexuellement après qu'ils auront réalisé les diverses activités contenues dans le jeu ITS: Stopper la transmission.

\section{Méthodologie}

Dans cette partie, nous présentons la démarche en deux volets: 1) les types de recherches privilégiés dans l'étude et les sujets qui font l'objet d'expérimentation, les instruments de mesure, la description du jeu éducatif en ligne ITS: Stopper la transmission ainsi que 2) le déroulement de l'expérimentation, les méthodes d'analyse et les considérations éthiques.

\subsection{Les types de recherches et les sujets}

Deux recherches ont été réalisées: recherche de processus (ou validation auprès d'experts) et recherche d'efficacité. En ce qui a trait à la recherche de processus, nous avons soumis ce jeu à des experts avant son expérimentation sur le terrain afin de nous assurer que le contenu du jeu favorisait le changement d'attitudes. Cette validation ou recherche de processus s'est appuyée sur la méthode Learner Verification and Revision (L.V.R.), processus d'évaluation formative qui permet d'améliorer un jeu pendant qu'il est encore en phase de réalisation (Kandaswany, Stolovitch et Thiagarajan, 1976; Caron-Bouchard et Renaud, 2001; Sauvé et Samson, 2004). Pour effectuer l'évaluation du contenu du jeu, 10 experts (3 professeurs de secondaire, 3 sexologues, 1 animateur de la vie étudiante, 1 éducateur à la santé, 2 ergonomes du Web) ont été consultés individuellement à l'aide d'une grille d'entrevue. Ils ont eu deux semaines pour naviguer dans le jeu et nous livrer, oralement et par écrit, leurs commentaires. Ces personnes ont été choisies pour leur expertise, tant du public cible, que de la thématique des infections transmissibles sexuellement et de l'ergonomie du jeu sur Internet. Elles ont évalué l'exactitude du contenu véhiculé par le jeu, la concordance entre les objectifs poursuivis et les contenus, la spécificité des objectifs, la complexité et le degré de difficulté du jeu pour la clientèle. Par la suite, le jeu a été modifié en fonction de cette évaluation.

Quant à la recherche sur l'efficacité, nous avons expérimenté le jeu éducatif en ligne auprès d'élèves du secondaire afin de cerner l'efficacité de ce jeu sur le changement d'attitudes. Un protocole de type pré et post-test simple groupe a été mis en place auprès de 173 élèves ( 95 garçons et 78 filles) de troisième secondaire, âgés 
de 13 à 14 ans, échantillonnés par grappes en raison de la difficulté à expérimenter dans les écoles. Ainsi, les participants ont été invités à prendre part à l'expérimentation en fonction d'un groupe classe d'une école. L'expérimentation s'est déroulée dans deux écoles, l'une à Laval, l'autre à Québec.

\subsection{Les instruments de mesure de l'expérimentation}

Une fois les instruments de mesure mis à l'essai auprès d'un échantillon restreint de la population cible (27 élèves), ces instruments ont été administrés aux 173 participants, à différents moments de l'expérimentation. Les élèves ont d'abord été amenés à remplir la Fiche d'inscription (sexe, âge, année scolaire dans laquelle le sujet est inscrit); ensuite, le Questionnaire d'attitudes sur infections transmissibles sexuellement, pour établir les attitudes préalables face aux infections à transmission sexuelle et, enfin, le Questionnaire post-expérimentation sur les attitudes des sujets par rapport aux infections à transmission sexuelle, pour identifier les apprentissages réalisés à l'aide du jeu en ligne.

Afin de répondre à l'objectif spécifique de l'étude, mesurer le degré de changement d'attitudes favorisé par un jeu éducatif en ligne envers les infections transmissibles sexuellement, nous avons utilisé 34 indicateurs de changement d'attitudes (Tableau 1) liés à trois composantes majeures explicatives de la mise en mouvement intérieure de l'individu: 1) sa prédisposition à agir, déterminée par sa perception des infections transmissibles sexuellement en général, la perception de sa propre sexualité, la perception de sa propre vulnérabilité aux infections transmissibles sexuellement, la perception de la gravité et des dangers des infections transmissibles sexuellement, ainsi que la perception de contrôle vis-à-vis de l'utilisation des condoms; 2) ses habitudes personnelles face à la sexualité et à la prévention et 3) la perception qu'il a de solutions accessibles et pratiques, s'exprimant d'une part par les gains ou bénéfices personnels et, d'autre part, par l'ampleur des obstacles à passer à l'action.

Tableau 1

Indicateurs et items d'analyse en termes d'attitudes

\begin{tabular}{|c|c|}
\hline Indicateurs & ltems \\
\hline \multicolumn{2}{|c|}{1 a) La prédisposition à agir } \\
\hline \multirow{5}{*}{$\begin{array}{l}\text { Votre perception } \\
\text { générale des } \\
\text { infections } \\
\text { transmissibles } \\
\text { sexuellement }\end{array}$} & $\begin{array}{l}\text { Je me sens libre de parler de sexualité et des infections transmissibles } \\
\text { sexuellement avec ma famille. }\end{array}$ \\
\hline & $\begin{array}{l}\text { Je me sens libre de parler de sexualité et des infections transmissibles } \\
\text { sexuellement avec mes ami(e)s. }\end{array}$ \\
\hline & $\begin{array}{l}\text { Je me sens libre de parler de sexualité et des infections transmissibles } \\
\text { sexuellement avec mon/ma partenaire. }\end{array}$ \\
\hline & $\begin{array}{l}\text { Je pense que mes amis ont tendance à séparer la relation amoureuse des } \\
\text { rapports sexuels. }\end{array}$ \\
\hline & $\begin{array}{l}\text { Je conseillerais à mes ami(e)s d'utiliser un condom lors d'un rapport sexuel oral } \\
\text { ou anal. }\end{array}$ \\
\hline
\end{tabular}


Renaud, L. et Sauvé, L. 681

\begin{tabular}{|c|c|}
\hline Indicateurs & Items \\
\hline \multirow{2}{*}{$\begin{array}{l}\text { Votre perception } \\
\text { quant à votre } \\
\text { propre sexualité }\end{array}$} & $\begin{array}{l}\text { Je serais capable de refuser une pratique sexuelle avec laquelle je ne suis pas à } \\
\text { l'aise. }\end{array}$ \\
\hline & Je serais capable de prendre l'initiative lors d'une relation sexuelle. \\
\hline \multirow{4}{*}{$\begin{array}{l}\text { La perception qu'a } \\
\text { l'individu de sa } \\
\text { propre vulnérabilité } \\
\text { aux infections } \\
\text { transmissibles } \\
\text { sexuellement }\end{array}$} & J'ai peur d'attraper une infection transmissible sexuellement. \\
\hline & J'ai peur d'attraper le virus du sida. \\
\hline & Je crois que je peux attraper une infection transmissible sexuellement. \\
\hline & Je crois que je suis vulnérable à une infection transmissible sexuellement. \\
\hline \multirow{7}{*}{$\begin{array}{l}\text { La perception de la } \\
\text { gravité et des } \\
\text { dangers des } \\
\text { infections } \\
\text { transmissibles } \\
\text { sexuellement }\end{array}$} & $\begin{array}{l}\text { Je crois que les infections transmissibles sexuellement sont une maladie } \\
\text { importante. }\end{array}$ \\
\hline & $\begin{array}{l}\text { Je crois que je ne pourrais plus faire mes occupations si j'avais une infection } \\
\text { transmissible sexuellement. }\end{array}$ \\
\hline & $\begin{array}{l}\text { Je crois que les infections transmissibles sexuellement pourraient me rendre } \\
\text { malade. }\end{array}$ \\
\hline & $\begin{array}{l}\text { Je crois que si je n'utilise pas les condoms, je peux attraper une infection } \\
\text { transmissible sexuellement. }\end{array}$ \\
\hline & Je connais l'importance de l'utilisation du condom dans toute relation sexuelle. \\
\hline & Je connais les risques possibles des relations sexuelles non protégées. \\
\hline & Je connais les conséquences de certaines infections à transmission sexuelle. \\
\hline \multirow{4}{*}{$\begin{array}{l}\text { Votre perception de } \\
\text { contrôle vis-à-vis } \\
\text { l'utilisation du } \\
\text { condom }\end{array}$} & $\begin{array}{l}\text { Compte tenu de ce que je vis, si j'avais à utiliser le condom, je me sentirais } \\
\text { capable d'acheter des condoms, sans gêne. }\end{array}$ \\
\hline & $\begin{array}{l}\text { Compte tenu de ce que je vis, si j'avais à utiliser le condom, je me sentirais } \\
\text { capable de surmonter ma gêne de parler du condom à mon (ma) partenaire. }\end{array}$ \\
\hline & $\begin{array}{l}\text { Compte tenu de ce que je vis, si j'avais à utiliser le condom, je me sentirais } \\
\text { capable de surmonter ma gêne d'installer un condom. }\end{array}$ \\
\hline & $\begin{array}{l}\text { Compte tenu de ce que je vis, si j'avais à utiliser le condom, je me sentirais } \\
\text { capable d'utiliser un condom même si un autre contraceptif est utilisé. }\end{array}$ \\
\hline
\end{tabular}

$1 \mathrm{~b})$ Les habitudes personnelles

Face à la sexualité Dans ma sexualité, il est important pour moi de partager mes sentiments, mes et à la prévention émotions avec mon/ma partenaire.

Dans ma sexualité, il est important pour moi de respecter mon/ma partenaire dans ses besoins, ses désirs.

Dans ma sexualité, il est important pour moi d'aider mon/ma partenaire à partager ses sentiments, ses émotions.

Éventuellement, je serais capable de dire à mon/ma partenaire comment il/elle peut me faire plaisir sexuellement.

Éventuellement, je serais capable de mettre le condom sans gêne pendant la relation sexuelle.

$1 \mathrm{c}$ ) La perception de solutions accessibles et pratiques

Gains personnels Porter le condom me protège contre les infections transmissibles sexuellement. L'abstinence me protège contre les infections transmissibles sexuellement.

Communiquer avec mon/ma partenaire peut m'aider à me protéger contre les infections transmissibles sexuellement.

Communiquer avec mon/ma partenaire peut m'aider à me protéger contre les infections transmissibles sexuellement.

Je serais capable de refuser une pratique sexuelle avec laquelle je ne suis pas à l'aise. 


\begin{tabular}{ll}
\hline Indicateurs & Items \\
\hline $\begin{array}{l}\text { Envergure des } \\
\text { obstacles à l'action }\end{array}$ & Je crois que l'achat des condoms est gênant. \\
& Je crois que l'utilisation du condom nous procure moins de sensations. \\
& Je crois que les condoms coûtent trop cher. \\
& Je crois que mon ami(e) va me juger si je lui demande d'utiliser le condom. \\
\hline
\end{tabular}

Nous avons construit une échelle de Likert à cinq items. Les questionnaires sur les attitudes sont auto-rapportés.

\subsection{Description du jeu ITS: Stopper la transmission}

Deux médecins praticiens se sont associés à l'équipe de recherche pour concevoir un jeu éducatif en ligne sur les infections transmissibles sexuellement, intitulé ITS: Stopper la transmission. Le VIH ne sera pas abordé dans cette étude, parce qu'il fait partie des ITSS. En effet, nous n'avons examiné que les maladies qui se transmettent lors de rapports sexuels oraux, anaux et vaginaux, comme la chlamydia et la gonorrhée.

Les 19 activités d'apprentissage du jeu ont pour objectif de modifier les attitudes des joueurs. Elles sont présentées sous la forme de questions ouvertes à réponse narrative incluant une rétroaction. De façon plus spécifique, 12 activités du jeu présentent des scénarios imprécis, mais délimités, d'une situation donnée; par exemple, Que réponds-tu à la personne qui te dis: «C'est à la mode de prendre des risques, à bas le condom?» Sept activités d'apprentissage offrent des démonstrations précises (correctes ou incorrectes); par exemple, une carte montre le verso d'un emballage de condom et le joueur est invité à dire si la date de péremption est dépassée ou non, ou encore, entre deux emballages de condom qu'on lui présente, il doit choisir le bon. Toutes les activités mettent le joueur en situation d'interaction avec les autres joueurs et proposent une rétroaction. Pour chaque bonne réponse, il reçoit un renforcement positif et, pour chaque erreur, une rétroaction explicative pour lui permettre de mieux «performer» la prochaine fois.

\subsection{Déroulement}

La recherche de processus ou validation auprès d'experts a été effectuée au printemps 2007, où les 10 experts ont été interrogés sur leur lieu de travail pendant environ 90 minutes. La plupart ont proposé quelques améliorations sur le plan de la structure du jeu. Quant au contenu, trois d'entre eux ont proposé de nouvelles informations et nous ont aidées à réviser le jeu.

Quant à la recherche d'efficacité, elle s'est déroulée de novembre 2007 à février 2008. Les instruments de pré-expérimentation ont été terminés une semaine avant l'expérimentation du jeu en classe. Deux séances de 60 minutes ont été consacrées au jeu. Les instruments de post-expérimentation ont été prêts une semaine après la pratique du jeu. 


\subsection{Méthode d'analyse des données}

Nous avons traité les données quantitatives et nous avons effectué des analyses statistiques à l'aide du logiciel SPSS (Statistical Package for Social Sciences). Un pré-test de l'instrument de l'échelle de Likert nous a permis de constater la consistance interne des items. Un a de Cronbach a été appliqué pour déterminer la cohérence interne des échelles de mesure.

L'analyse des données provenant de l'échelle de Likert a été réalisée de la façon suivante. Nous avons calculé la moyenne des données de l'échelle ordinale (Likert) en la considérant comme une échelle métrique, c'est-à-dire que nous supposions que les écarts seraient constants entre les différentes modalités. Par conséquent, les variables quantitatives ont été appliquées en attribuant un nombre arbitraire à chaque réponse (par exemple, 1 pour désaccord total et 5 pour tout à fait d'accord). Nous avons obtenu une note moyenne. Les résultats ont fait l'objet d'un $\chi^{2}$ (khi carré) qui mesure l'association entre l'item au pré-test et celui du post-test, et ils sont statistiquement significatifs $(p \leq 0,001)$.

\subsection{Considérations éthiques}

Tous les participants ont signé un formulaire de consentement. Dans le cas des élèves, la signature des parents ou des tuteurs était requise. Le formulaire de consentement comportait quatre parties: l'identification des membres de l'équipe, une brève description du projet de recherche, le déroulement de l'expérience avec toutes les étapes des divers tests et une section sur les principes éthiques de confidentialité des données, sans aucune possibilité de retracer le sujet d'aucune manière. Nous avons attribué un numéro à chaque élève afin de ne pas révéler son nom lors de la manipulation des questionnaires et lors des analyses. Les résultats de la validation ont été discutés avec les divers professionnels afin de réajuster le jeu. Quant aux résultats qui portent sur l'efficacité du jeu, ils ont été abordés avec le corps professoral et l'animatrice en sexualité des écoles concernées.

\section{Résultats}

Dans cette section, nous présentons deux types de résultats: ceux en lien avec la validation du jeu et ceux qui portent sur le changement d'attitudes chez les élèves qui ont expérimenté le jeu éducatif en ligne.

\section{1 Évaluation de processus (ou validation) auprès des experts}

Bien que le jeu ait été conçu par des médecins qui avaient une expertise de contenu et non une expertise pédagogique, il s'avérait important de faire valider le jeu par des experts afin de s'assurer de la justesse du contenu, de l'adéquation du jeu au public cible, de l'aspect visuel et du degré de difficulté des activités.

Sur le plan des contenus, les experts considèrent que le titre du jeu est explicite par rapport au contenu, et que les informations véhiculées dans les activités sur 
les infections transmissibles sexuellement sont exactes du point de vue scientifique. Ils ont constaté toutefois que certains éléments clés de l'éducation à la sexualité sont absents du jeu: notamment, la notion de plaisir. Ils précisent que le contenu du jeu constitue un outil pertinent pour la vérification des connaissances davantage que pour leur acquisition par les étudiants au postsecondaire d'une part et, d'autre part, que le contenu est plus ou moins éloigné des préoccupations, croyances et représentations des élèves du secondaire.

Par rapport au public cible, les experts concluent que le jeu ITS: Stopper la transmission vise des objectifs ambitieux et cible un public trop large: de la troisième secondaire aux deux premières années universitaires. À leur avis, le contenu du jeu est plutôt approprié pour les étudiants du postsecondaire, et particulièrement pour ceux qui étudient en sexologie ou en médecine.

En ce qui a trait à l'aspect visuel du jeu, peu de commentaires ont été formulés. Tout en trouvant la structure du jeu intéressante et motivante, les experts jugent que le plateau de jeu (couleur, image) rejoint plus la clientèle du postsecondaire et sera jugé moins attrayant par les adolescents. Par ailleurs, ils notent que les activités d'apprentissage n'offrent pas assez de contenu multimédia et dynamique. Ils considèrent que toutes les activités devraient offrir ce type de contenu pour rejoindre les adolescents.

En ce qui a trait à la complexité et au degré de difficulté des activités d'apprentissage du jeu pour les élèves du secondaire et les jeunes adultes, profanes en matière d'infection transmissible sexuellement, il est clair, toujours selon les experts, que le jeu exigerait une adaptation sur le plan du langage et du traitement médiatique des activités d'apprentissage. D’où leur suggestion de retravailler la majorité des activités

Sur les 79 activités proposées dans le jeu (dont 19 conçues explicitement pour agir sur les attitudes), seulement 15 n'ont pas fait l'objet de remarques quant à leur pertinence ou à leur formulation. D'après les experts, certaines des activités d'apprentissage proposées par le jeu ne sont pas classées dans la bonne catégorie. De plus, le vocabulaire n'est pas toujours uniforme. Ils jugent les liens entre les questions et les illustrations de cinq activités peu adaptés et susceptibles de porter à confusion. Quant aux vidéos proposées, qui s'adressent davantage au public du postsecondaire, les experts demandent qu'elles soient retravaillées pour les élèves du secondaire.

En ce qui concerne l'intégration du jeu dans la grille horaire, tous les experts considèrent que la durée du jeu permet son utilisation à l'intérieur d'une période de classe, et qu'il est facile d'y rejouer à l'extérieur de la classe, pour les élèves intéressés.

Toutes les corrections suggérées par les experts sur les activités d'apprentissage ont été prises en compte et une nouvelle version du jeu a été élaborée. 


\subsection{Principaux résultats d'efficacité sur les attitudes des joueurs}

La présentation des résultats en lien avec le changement d'attitudes sera faite à travers le compte-rendu de diverses perceptions: perception générale, perception de sa propre sexualité, de sa vulnérabilité, de la gravité et des dangers, perception de contrôle, perception de solutions accessibles et perception des pratiques en termes de gains personnels et d'obstacles à l'action. Le tableau 2 présente les résultats les plus significatifs de l'étude. Les lecteurs qui désirent connaître tous les résultats peuvent consulter le rapport complet disponible sur le site du Carrefour virtuel de jeux éducatifs (http://carrefour-jeux.savie.ca).

\section{Tableau 2}

Comparaison de résultats en pré-test et post-test au sujet de quelques items à l'égard des ITS

\begin{tabular}{|c|c|c|c|c|}
\hline $\begin{array}{l}\text { Indicateurs } \\
\text { (a de Cronbach*) }\end{array}$ & Item & $\begin{array}{l}\text { En accord } \\
\text { en pré- } \\
\text { test \% }\end{array}$ & $\begin{array}{l}\text { En accord } \\
\text { en post- } \\
\text { test } \%\end{array}$ & $\chi^{2}$ \\
\hline \multirow{4}{*}{$\begin{array}{l}\text { Votre perception } \\
\text { générale des } \\
\text { infections } \\
\text { transmissibles } \\
\text { sexuellement } \\
(0,509)\end{array}$} & $\begin{array}{l}\text { Je me sens libre de parler de sexualité et } \\
\text { des ITS avec ma famille. }\end{array}$ & 24,4 & 48,8 & $\begin{array}{c}70,86 \\
(p \leq 0,000)\end{array}$ \\
\hline & $\begin{array}{l}\text { Je me sens libre de parler de sexualité et } \\
\text { des ITS avec mes ami(e)s. }\end{array}$ & 63,3 & 75,5 & $\begin{array}{c}78,77 \\
(p \leq 0,000)\end{array}$ \\
\hline & $\begin{array}{l}\text { Je me sens libre de parler de sexualité et } \\
\text { des ITS avec mon/ma partenaire. }\end{array}$ & 70,9 & 82,6 & $\begin{array}{c}78,75 \\
(p \leq 0,000)\end{array}$ \\
\hline & $\begin{array}{l}\text { Je conseillerais à mes ami(e)s d'utiliser } \\
\text { un condom lors d'un rapport sexuel. }\end{array}$ & 83,3 & 88,8 & $\begin{array}{c}60,68 \\
(p \leq 0,000)\end{array}$ \\
\hline \multirow{2}{*}{$\begin{array}{l}\text { Votre perception } \\
\text { quant à } \\
\text { votre propre sexualité } \\
(0,374)\end{array}$} & $\begin{array}{l}\text { Je serais capable de refuser une pratique } \\
\text { sexuelle avec laquelle je ne suis pas à } \\
\text { l'aise. }\end{array}$ & 71,8 & 79,6 & $\begin{array}{c}68,87 \\
(p \leq 0,000)\end{array}$ \\
\hline & $\begin{array}{l}\text { Je serais capable de prendre l'initiative } \\
\text { lors d'une relation sexuelle. }\end{array}$ & 91,9 & 89,7 & $\begin{array}{c}47,69 \\
(p \leq 0,000)\end{array}$ \\
\hline $\begin{array}{l}\text { La perception qu'a } \\
\text { l'individu de sa } \\
\text { propre vulnérabilité } \\
\text { aux infections }\end{array}$ & Je crois que je suis vulnérable à une ITS. & 67,7 & 64,4 & $\begin{array}{c}39,49 \\
(p \leq 0,001)\end{array}$ \\
\hline $\begin{array}{l}\text { transmissibles } \\
\text { sexuellement } \\
(0,737)\end{array}$ & J'ai peur d'attraper une ITS. & 30,7 & 45,4 & $\begin{array}{c}49,12 \\
(p \leq 0,000)\end{array}$ \\
\hline $\begin{array}{l}\text { La perception de la } \\
\text { gravité et des } \\
\text { dangers des } \\
\text { infections } \\
\text { transmissibles } \\
\text { sexuellement } \\
(0,612)\end{array}$ & $\begin{array}{l}\text { Je crois que je ne pourrais plus faire mes } \\
\text { occupations si j'avais une ITS. }\end{array}$ & 38,2 & 40,4 & $\begin{array}{c}23,00 \\
(p \leq 0,000)\end{array}$ \\
\hline
\end{tabular}




\begin{tabular}{|c|c|c|c|c|}
\hline $\begin{array}{l}\text { Indicateurs } \\
(\alpha \text { de Cronbach*) }\end{array}$ & ltem & $\begin{array}{l}\text { En accord } \\
\text { en pré- } \\
\text { test } \%\end{array}$ & $\begin{array}{l}\text { En accord } \\
\text { en post- } \\
\text { test } \%\end{array}$ & $\chi^{2}$ \\
\hline $\begin{array}{l}\text { Votre perception de } \\
\text { contrôle vis-à-vis } \\
\text { l'utilisation du } \\
\text { condom } \\
(0,843)\end{array}$ & $\begin{array}{l}\text { Je me sentirais capable d'acheter des } \\
\text { condoms sans gêne. }\end{array}$ & 58,4 & 64 & $\begin{array}{c}83,24 \\
(p \leq 0,000)\end{array}$ \\
\hline \multirow[t]{2}{*}{$\begin{array}{l}\text { Gains personnels } \\
(0,260)\end{array}$} & L'abstinence me protège contre les ITS. & 58,8 & 63,5 & $\begin{array}{c}42,68 \\
(p \leq 0,000)\end{array}$ \\
\hline & $\begin{array}{l}\text { Communiquer avec mon/ma partenaire } \\
\text { peut m'aider à me protéger contre les } \\
\text { ITS. }\end{array}$ & 58,8 & 63,5 & $\begin{array}{c}35,74 \\
(p \leq 0,003)\end{array}$ \\
\hline \multirow{2}{*}{$\begin{array}{l}\text { Envergure des } \\
\text { obstacles à l'action } \\
(0,752)\end{array}$} & $\begin{array}{l}\text { Je crois que l'achat des condoms est } \\
\text { gênant. }\end{array}$ & 41,1 & 37,6 & $\begin{array}{c}62,19 \\
(p \leq 0,000)\end{array}$ \\
\hline & $\begin{array}{l}\text { Je crois que l'utilisation du condom me } \\
\text { procure moins de sensation. }\end{array}$ & 24,7 & 22,3 & $\begin{array}{c}52,96 \\
(p \leq 0,000)\end{array}$ \\
\hline
\end{tabular}

* L'a de Cronbach a été calculé sur l'ensemble des items d'un indicateur (voir tableau 1). Le tableau présente quelques items par indicateur.

La perception générale des jeunes à l'égard des infections transmissibles sexuellement s'est améliorée avec le jeu. Les jeunes disent qu'ils se sentent plus libres de parler de leur sexualité et des infections transmissibles sexuellement avec leurs familles après l'expérimentation ( $48,8 \%$, contre $24,4 \%$ avant); ils se sentent également plus libres de parler de sexualité avec leurs ami(e)s (75,5\% contre $63,3 \%$ avant), avec leur partenaire ( $82,6 \%$ contre $70,9 \%$ avant); enfin, ils conseilleraient dans une mesure légèrement plus importante à leurs ami(e)s d'utiliser un condom lors d'un rapport sexuel (88,8\% contre $83,3 \%$ avant).

Quant à la perception de leur propre sexualité, le jeu aurait permis aux participants d'être moins naïfs et plus sincères. Dans une proportion de 79,6\%, les élèves sont d'avis qu'ils seraient capables de refuser une pratique sexuelle avec laquelle ils ne sont pas à l'aise, contre 71,8\% avant l'expérimentation. Par ailleurs, 91,9\% des jeunes se disent capables de prendre l'initiative lors d'une relation sexuelle, contre $89,7 \%$ avant l'expérimentation.

À propos de la perception, par l'individu, de sa propre vulnérabilité aux infections transmissibles sexuellement, nous remarquons que le jeu a permis d'accroître le sentiment de vulnérabilité des étudiants en regard de la possibilité de contracter une infection transmissible sexuellement. En effet, le participant croit davantage qu'il peut attraper une infection transmissible sexuellement après avoir joué le jeu qu'avant $(67,7 \%$ contre $64,4 \%)$. De plus, il croit être davantage vulnérable à une infection transmissible sexuellement après l'expérimentation qu'avant; ici, les résultats montrent une augmentation de la peur d'attraper une infection transmissible sexuellement (45,4\% après le jeu contre $30,7 \%$ avant).

En ce qui concerne la perception de la gravité et des dangers des infections 
transmissibles sexuellement, soulignons un léger changement à l'égard de la difficulté à poursuivre leurs activités habituelles : après l'expérimentation, 40,4\% d'entre eux croient qu'ils ne pourraient plus maintenir leurs activités s'ils avaient une infection transmissible sexuellement, contre 38,2\% avant.

En regard de la perception de contrôle vis-à-vis de l'utilisation du condom, la seule différence significative entre les attitudes des jeunes à ce sujet, avant et après l'expérimentation, concerne l'achat des condoms: $64 \%$ d'entre eux disent se sentir capables d'acheter des condoms sans gêne après le jeu, contre 58,4\% avant.

$\mathrm{Au}$ sujet des solutions accessibles aux jeunes et des pratiques (gains personnels), nous remarquons une légère amélioration de leur perception que l'abstinence les protège contre les infections transmissibles sexuellement $(63,5 \%$ sont très en accord et en accord avec cette affirmation après l'expérimentation, contre 58,8\% avant), et on observe les mêmes résultats pour l'énoncé: Communiquer avec mon partenaire peut m'aider à me protéger contre les infections transmissibles sexuellement. Par ailleurs, la perception du port du condom comme solution favorable n'a pas été augmentée avec le jeu.

Finalement, en ce qui concerne la perception de solutions accessibles et celle des pratiques (l'ampleur des obstacles à l'action), nous remarquons que le jeu permet d'améliorer la perception de la possibilité de contrer les obstacles. L'achat des condoms est moins gênant après l'expérimentation selon 37,6\% après l'expérimentation, contre $41,1 \%$ avant; enfin, l'utilisation du condom est perçue comme un moyen qui limite la sensation de plaisir selon $24,7 \%$ après l'expérimentation, contre $22,3 \%$ avant.

\section{Discussion des résultats}

La recherche de validation du jeu par des experts a permis de constater que ITS: Stopper la transmission, tel qu'il est présenté et créé par les médecins, s'adresse davantage à un public universitaire dans le cadre de la vérification d'acquis. Pour adapter le jeu aux élèves du secondaire, plusieurs recommandations et suggestions ont été formulées. Les experts pédagogiques consultés ont grandement participé en reformulant les questions afin de mieux les adapter à un public plus jeune.

Par ailleurs, par le biais des intervenants et des enseignants, le milieu scolaire a bien accueilli ce jeu éducatif en ligne. En effet, cette stratégie d'enseignement s'utilise bien en classe, à l'intérieur d'une période de cours. Le jeu ne nécessite pas d'expertise spéciale de la part de l'enseignant. De plus, l'élève peut y jouer de nouveau à la maison, seul ou avec ses amis, s'il en ressent le besoin.

Les résultats de la validation confirment plusieurs avantages attribués aux jeux éducatifs en ligne par rapport à des interventions en face à face (Kanuga, 2004) : ces derniers peuvent être anonymes, répétés en tout temps. Ils permettent également un apprentissage individualisé et actif, selon le mode d'utilisation qui en est fait (Barak et Fisher, 2001). Les jeux peuvent aussi fournir des contenus d'apprentissage qui seraient difficiles ou embarrassants à aborder en face à face, 
comme le souligne Ochs (1994), qui cite l'exemple de la thérapie sexuelle. Enfin, les jeux favorisent une dissémination en ligne plus rapide et relativement bon marché (Barak et Fisher, 2001).

Cette validation amène également les chercheuses à discuter de la nécessité d'une expertise, tant sur le plan pédagogique que sur celui du public cible. Les environnements de conception de jeux éducatifs mis au point par le Centre d'expertise et de recherche sur l'apprentissage à vie (SAVIE) sont offerts pour que des experts, désireux de réaliser des activités dans le domaine de la santé, puissent développer un jeu éducatif en quelques heures seulement à partir du Web. Cependant, il ne suffit pas de posséder des connaissances scientifiques pertinentes pour concevoir un jeu adapté aux jeunes. Les représentations, le langage, les préoccupations du public cible, le cheminement pédagogique et le contexte du jeu doivent également être pris en compte pour que le contenu soit réellement adapté et vecteur de changements d'attitudes. Grâce à la recherche de validation, le jeu ITS: Stopper la transmission a été amélioré en tenant compte des commentaires des experts.

En santé publique, la promotion de la santé sexuelle est un défi de taille. Comment rejoindre les jeunes et quelle stratégie permettrait de générer un changement d'attitudes? Nous les avons rejoints en milieu scolaire et nous avons utilisé un jeu éducatif en ligne. Cette stratégie est-elle efficace pour modifier les attitudes des jeunes à l'égard des infections transmissibles sexuellement? Nos résultats montrent une légère amélioration des attitudes des joueurs: nous avons effectivement noté 1) une amélioration de la perception générale des jeunes à l'égard des infections transmissibles sexuellement, 2) une perception accrue de leur vulnérabilité, 3) une meilleure perception de la gravité des conséquences, 4) un fort sentiment de contrôle, 5) une perception accrue de leur capacité à contrer les obstacles pour passer à l'action. Ainsi, les élèves ont développé des attitudes plus favorables à des actions en lien avec les infections transmissibles sexuellement (négocier le port du condom, réduire la gêne à l'achat de ce dernier). Même s'ils se montraient favorables à l'idée de pratiquer le sexe "sécuritaire", cela ne les a pas conduits nécessairement à l'utilisation de préservatifs, comme l'indiquent les résultats sur la perception du port du condom, qui ne s'est pas améliorée. De plus, plusieurs items ne semblent pas avoir donné l'effet escompté, notamment ceux liés à la perception de sa propre sexualité.

Ces résultats découlent-ils de notre instrument de mesure ou de la complexité du sujet? Selon Potter (2001), les variables d'attitudes envers la sexualité sont difficilement mesurables, puisque leurs significations sont complexes et que cette complexité est résumée et traduite par des items transposés dans une échelle qui se calcule, au final, par une valeur numérique. L'élaboration de notre instrument repose sur le modèle de croyances relatives à la santé et met de l'avant trois composantes majeures explicatives des attitudes: 1) la prédisposition des sujets à agir, 2) les habitudes personnelles face à la sexualité et à la prévention et 3) la perception 
de solutions accessibles et pratiques. Bien que notre instrument de mesure fasse l'objet d'une validité interne, y aurait-il eu certaines dimensions dont le construit aurait pu être plus adéquat et pertinent pour mesurer, identifier et clarifier les perceptions et attitudes des jeunes à l'égard de la sexualité et des infections transmissibles sexuellement?

Certains auteurs considèrent que le thème de la sexualité et des infections transmissibles sexuellement abordé par le jeu éducatif en ligne est un sujet complexe puisqu'il est fortement lié à des valeurs sociétales et personnelles. Est-il préférable et désirable d'observer une augmentation ou une diminution dans une attitude particulière? Par exemple, il n'est pas évident de savoir si une attitude «conservatrice» (attendre le mariage pour faire l'amour) ou «libérale» à l'égard de la sexualité est préférable et désirable, dans la perspective d'une définition holistique de la santé sexuelle (Organisation mondiale de la Santé, 2002; Alemi et collab., 1989; Bailey et collab., 2009; Roberto et collab., 2007). Selon nos résultats, l'utilisation du jeu interactif en ligne a permis aux jeunes de développer des attitudes favorables à la communication interpersonnelle lors de la négociation du condom et de son achat; toutefois, nos résultats montrent que le jeu ne les amène pas à être prêts à utiliser le condom. Ces résultats sont-ils en lien avec leur groupe d'âge (13-14 ans)? Pour eux, utiliser le condom signifierait-il se projeter dans l'avenir et avoir des relations sexuelles?

Quant à la perception de leur propre sexualité, le jeu éducatif en ligne amène les élèves à répondre avec plus de prudence. Se pourrait-il que le jeu montre aux élèves la complexité des relations humaines et la difficulté à négocier? Pourrionsnous formuler l'hypothèse que les réponses au pré-test ont été faites sur un mode plus naï et que le jeu ait amené les participants à être plus sensibles et plus concernés par ce qu'ils feraient réellement, et donc plus sincères dans les réponses après le jeu (Kann, 1987; Roberto et collab., 2007; Bailey et collab., 2009) ? Nous croyons que les élèves ont été sensibilisés à la problématique des infections transmissibles sexuellement et qu'ils ont davantage réfléchi qu'au pré-test pour répondre au post-test, comme l'ont également constaté Green et Kreuter (2000) dans leur étude. Cette hypothèse devra être vérifiée dans une prochaine étude.

\section{Conclusion}

La présente recherche avait pour objectif d'évaluer l'efficacité d'un jeu éducatif en ligne conçu par des enseignants et des praticiens de la santé pour la prévention et la promotion de la santé, en matière de changements d'attitudes envers la santé sexuelle, auprès d'élèves de 13 et 14 ans.

Les résultats de notre étude montrent que la recherche du processus pour valider le jeu ITS: Stopper la transmission est nécessaire et préalable à l'évaluation de son efficacité. Cette validation nous assure minimalement que le jeu convient aux réalités des jeunes. Quant aux résultats de l'évaluation d'efficacité du jeu sur le plan des attitudes, ils nous indiquent qu'une certaine modification des attitudes est 
possible en jouant à un jeu éducatif en ligne. Dans la recherche d'activités diversifiées pour amener les élèves à prendre leur santé en main, le jeu éducatif en ligne joué dans un contexte scolaire peut offrir des apprentissages affectifs pertinents.

Cependant, certaines limites de cette étude ne nous permettent pas de généraliser les résultats. Nous avons opté pour l'examen des attitudes, c'est-à-dire l'examen des dispositions mentales à l'agir, plutôt que pour l'observation du comportement lui-même. Ce choix est lié aux difficultés inhérentes à la problématique des relations sexuelles, à leur caractère intime et privé. De plus, le choix des répondants dans un contexte d'expérimentation dans les écoles secondaires ne nous permettait pas d'obtenir un échantillon probabiliste. Afin d'atténuer l'effet de cette dimension sur nos résultats, nous avons vérifié si notre échantillon correspondait à notre population de la génération numérique. Les résultats nous indiquent que notre échantillon non probabiliste est représentatif des caractéristiques de notre population sur le plan des technologies de l'information et de la communication (TIC) ainsi que des jeux. Enfin, le temps imparti pour expérimenter le jeu, deux périodes de classe, ne semble pas suffisant pour permettre aux répondants de réaliser, au moins deux fois, les activités de jeu de rôle, ce qui aurait pu influencer différemment les résultats de cette étude. Il faut rappeler que c'est le hasard qui détermine les activités réalisées par les répondants ainsi que le nombre de fois que chaque joueur les réalisera pendant l'expérimentation.

Finalement, les résultats de cette étude nous permettent de suggérer quelques recommandations:

- expérimenter de nouveau le jeu sur une période plus longue, c'est-à-dire sur plus de deux périodes de classe, afin de vérifier si la répétition de ces activités affectera d'une façon plus importante le changement d'attitudes;

- réaliser l'expérimentation auprès de jeunes plus âgés et comparer les résultats entre les deux cohortes en fonction de l'âge;

- développer d'autres mesures d'attitudes afin de comparer les résultats et d'en discuter la sensibilité;

- expérimenter nos instruments de mesure avec d'autres jeux sur d'autres thématiques en santé auprès des jeunes de 13 et 14 ans.

ENGLISH TITLE - On-line educational game: validation by the experts and evaluation of the impact of the game on the attitudes of the young people towards the sexual health

SUMMARY - This article shows 1 - the interest and the pertinence of a multimedia educational game using Web, the objective of which is to intervene on the attitudes towards sexual health of youth; 2 - the process research (validation) using 10 experts examining the pertinence of the contents, the adequacy of the game for the target audience, the visual aspect and the degree of difficulty of the activities and 3 - the impact research (pre and post-test) using a questionnaire containing 34 indicators of attitudinal change towards sexual health of 173 youth aged 13 to 14 years old. The process evaluation shows that the game is appropriate for the realities of the youth involved. The results of game's impact show an improvement of attitudes towards sexual health.

KEY WORDS - educational game on line, sexual health, health education, attitude, young. 
Título - Validación por expertos y evaluación de la eficiencia de un juego educativo en línea sobre las actitudes de los jóvenes de 13 y 14 años respecto a la salud sexual

RESUMEN - Este artículo trata de la pertinencia de un juego educativo en línea que interviene en las actitudes respecto a la salud sexual. La investigación, controlada por diez expertos, permite examinar, entre otros, la exactitud del contenido y la adecuación del juego al público que pretende deservir. En cuanto a la eficiencia, tiene por objetivo determinar, por medio de un cuestionario (pre-post test) que consta de 34 indicadores, las actitudes de 173 jóvenes de 13 y 14 años respecto a la salud sexual. La evaluación del proceso evidencia la adecuación del juego a la realidad de los jóvenes. Con relación a la eficiencia, los resultados indican una mejora de las actitudes con respecto a la salud sexual.

PALABRAS CLAVES - juego educativo en línea, salud sexual, educación a la salud, actitudes, jóvenes.

\section{Références}

*Alemi, F., Cherry, F. et Meffert, G. (1989). Rehearsing decisions may help teenagers: an evaluation of a simulation game. Computers in biology \& medicine, 19(4), 283-290.

Bailey, J.-V., Murray, E., Rait, G., Mercer, C., Morris, R.-W., Peacock, R., Cassell, J. et Nazareth, I. (2009). Interactive computer-based interventions for sexual health promotion. Cochrane database of systematic reviews, Issue 2.

Baldaro, B., Tuozzi, G., Codispoti, M. et Montebarocci, O. (2004). Aggressive and nonviolent videogames: short-term psychological and cardiovascular effects on habitual players. Stress and health, 20, 203-208.

Barab, S. A., Thomas, M., Dodge, T., Carteaux, R. et Tuzun, H. (2005). Making learning fun. Educational technology research and development, 53(1), 86-107.

Barak, A. et Fisher, W.-A. (2001). Toward an Internet-driven, theoretically-based, innovative approach to sex education. Journal of sex research, 38(4), 324-332.

Bijker, M., Van Buuren, H. et Wynants, G. (2006). A comparative study of the effects of motivational and attitudinal factors on studying statistics. Proceeding of $7^{\text {th }}$ International Conference on Teaching Statistics. Salvador, Bahia, Brazil: International Association for Statistical Education.

Bottino, R.-M., Ferlino, L., Ott, M. et Tavella, M. (2007). Developing strategic and reasoning abilities with computer games at primary school level. Computers and education, 49(4), 1272-1286.

*Bowen, A., Horvath, K. et Williams, M. (2007). A randomized control trial of Internetdelivered HIV prevention targeting rural MSM. Health education research, 22(1), 120-127.

Caron-Bouchard, M. et Renaud, L. (2001). Pour mieux réussir vos communications en promotion de la santé. Montréal, Québec: Institut national de santé publique du Québec.

Centre francophone d'informatisation des organisations - CÉFRIO - (2009). Génération C: Les 12-24 ans - Moteurs de transformation des organisations. Rapport synthèse, Montréal, Québec, Canada.

Chauvin C. (2001). Le kit de formation. Paris, France: ESF éditeur. 
${ }^{*}$ Conseil des ministres de l'Éducation du Canada (2003). Étude sur les jeunes, la santé sexuelle, le VIH et le sida au Canada. Facteurs influant sur les connaissances, les attitudes et les comportements. Ottawa, ministère de l'Éducation, Canada.

${ }^{\star}$ Davidovich, U., de Wit, J. et Stroebe, W. (2006). Using the Internet to reduce risk of HIV infection in steady relationships. A randomized controlled trial of a tailored intervention for gay men. Dans Davidovich E. (Dir.): Liaisons dangereuses-HIV Risk Behavior and Prevention in Steady Gay Relationships. Amsterdam, Pays-Bas: Roel \& Uitgeefprojecten: 95-122.

${ }^{\star}$ Evans, A.-E., Edmundson-Drane, E.-W. et Harris, K.-K. (2000). Computer-assisted instruction: an effective instructional method for HIV prevention education? Journal of adolescent health, 26(4), 244-251.

Garris, R., Ahlers, R. et Driskell, J.-E. (2002). Games, motivation, and learning: a research and practice model. Simulation and gaming, 33(4), 441-467.

Gauthier, C., Mellouki, M., Simard, D., Bissonnette, S. et Richard, M. (2004). Interventions pédagogiques efficaces et réussite scolaire des élèves provenant de milieux défavorisés. Une revue de littérature. Rapport de recherche préparé pour le Fonds québécois de la recherche sur la société et la culture. Québec, Québec: Université Laval.

Gouvernement du Québec (2008). Portrait des infections transmissibles sexuellement et par le sang (ITSS) au Québec - Année 2007 (et projections 2008). Québec, Québec: Gouvernement du Québec.

Green, L.-W. et Kreuter, M.-W. (2000). Health promotion planning: an educational and environmental approach. Toronto, Ontario: Mayfield Publishing Company.

Hornung, R.-L., Lennon, P.-A., Garrett, J.-M., DeVellis, R.-F., Weinberg, P.-D. et Strecher, V.-J. (2000). Interactive computer technology for skin cancer prevention targeting children. American journal of preventive medicine, 18(1), 69-76.

${ }^{\star} J$ Jenkins, P.-R., Jenkins, R.-A., Nannis, E.-D., McKee, K.-T., Jr. et Temoshok, L.-R. (2000). Reducing risk of sexually transmitted disease and human immunodeficiency virus infection in a military STD clinic: evaluation of a randomized preventive intervention trial. Clinical infectious diseases, 30(4), 730-735.

Kandaswany, S., Stolovitch, H.-D. et Thiagarajan, S. (1976). Learner verification and revision: an experimental comparison of two methods. Educational technology research and development, 24(3), 316-328.

${ }^{\star}$ Kann, L.-K. (1987). Effects of computer-assisted instruction on selected interaction skills related to responsible sexuality. Journal of school health, 57(7), 282-287.

Kanuga, M. et Rosenfeld, W.-.D. (2004). Adolescent sexuality and the internet: the good, the bad, and the URL. Journal of pediatric \& adolescent gynecology, 17(2), 117-124.

${ }^{\star}$ Keine, S.-M. et Barta, W.-D. (2006). A brief individualized computer-delivered sexual risk reduction intervention increases HIV/AIDS preventive behavior. Journal of adolescent health, 39(3), 404-410.

Lacroix, C. et Cloutier, R. (2006). Ça s'exprime: la santé sexuelle ça se protège. Fascicule No 4, hiver. Québec, Québec: Gouvernement du Québec.

(2004). Le Petit Larousse illustré. Paris, France: Édition Larousse. 
Lieberman, D. A. (2001). Management of chronic pediatric diseases with interactive health games: theory and research findings. Journal of ambulatory care management, 24(1), 26-38, 103-120.

Lieberman, D. A. (1997). Interactive video games for health promotion: effects on knowledge, self-efficacy, social support, and health. Dans R. L. Street, W. R. Gold et T. Manning (Dir.): Health promotion and interactive technology: theoretical applications and future directions. Mahwah, New-Jersey: Lawrence Erlbaum.

${ }^{*}$ Mikolajczak, J., Kok, G. et Hospers, H.-J. (2008). Queermasters: developing a theory and evidence-based internet hiv-prevention intervention to promote hiv-testing among men who have sex with men, Applied Psychology: an international review, 57(4), 681697.

Mucchielli, A. (2002). Dictionnaire des méthodes qualitatives en sciences humaines et sociales, Paris, France: Armand Colin.

Oblingner, D. G. et Obligner, J. L. (2005). Educating the net generation. Raleigh, North Carolina, North Carolina State University: Educause, Center for Teaching Excellence.

Ochs, E.-P, Meana, M., Pare, L., Mah, K. et Binik, Y.-M. (1994). Learning about sex outside the gutter: attitudes toward a computer sex-expert system. Journal of sex \& marital therapy, 20(2), 86-102.

Organisation mondiale de la Santé (2002). Defining sexual health. OMS, Genève, Suisse, Département de santé reproductive.

Petty, R., Cacioppo, J. et Heesacker, M. (1981). Effects of rhetorical questions on persuasion: a cognitive response analysis. Journal of personality and social psychology, 40(3), 432-440.

Potter, J. (2001). Unfolding discourse analysis. Dans M. Wetherell, S. Taylor et S.-J. Yates (Dir.). Discourse theory and practice. London: Thousand Oaks, New Delhi: Sage, 198209.

Prensky, M. (2001). Digital game-based learning. New York, New York: McGraw-Hill.

Prensky, M. (2005). Engage me or enrage me: What today's learners demand. EDUCAUSE review, 40(5), 60-64.

Rassin, M., Gutman, Y. et Silner, D. (2004). Developing a computer game to prepare children for surgery. AORN journal, 80(6), 1099-1102.

${ }^{\star}$ Read, S., Miller, L., Appleby, P., Nwosu, M., Reynaldo, S. et Lauren, A. (2006). Socially optimized learning in a virtual environment: reducing risky sexual behavior among men who have sex with men. Human communication research, 32(1), 1-34.

Renaud, L. et Sauvé, L. (1990). Simulation et jeu de simulation: outils éducatifs appliqués à la santé. Montréal, Québec: Édition Agence d'Arc.

${ }^{\star}$ Roberto, A., Zimmerman, R., Carlyle, K., Abner, E., Cupp, P. et Hansen, G. (2007). The effects of a computer-based pregnancy, STD, and HIV prevention intervention: a nine-school trial. Health communication, 21(2), 115-124.

Sauvé, L., Renaud, L., Kaufman, D., Samson, D., Bluteau-Doré, V., Dumais, C., Bujold, P., Kaszap, M. et Isabelle, C. (2005). Revue systématique des écrits (1998-2004) sur les fondements conceptuels du jeu, de la simulation et du jeu de simulation. Québec, 
Québec: Simulations and Advanced Gaming Environments for learning project (SAGE) et Centre d'expertise et de recherche sur l'apprentissage à vie (SAVIE).

Sauvé, L. et Samson, D. (2004). Rapport d'évaluation de la coquille générique du Jeu de l'oie du projet «Jeux génériques: multiplicateurs de contenu multimédia éducatif canadien sur l'inforoute». Québec, Québec: Centre d'expertise et de recherche sur l'apprentissage à vie (SAVIE) et Fonds Inukshuk Sans-fil inc..

Tingstrom, D. H., Sterling-Turner, H. et Wilczynski, S.-M. (2006). The good behavior game: 1969-2002. Behavior modification, 30(2), 225-253.

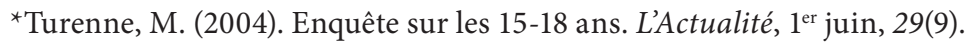

${ }^{*}$ Van Laar, S.-T. (2000). Internet-based cognitive restructuring: Effects on adolescent girls' contraceptive beliefs. Thèse de doctorat inédite, Arizona State University, Phoenix, Arizona.

Madame Lise Renaud est professeure titulaire au département de communication sociale et publique de l'Université du Québec à Montréal, et directrice de ComSanté, Centre de recherche sur les communications et la santé. Quant à madame Louise Sauvé, elle est professeure titulaire à TÉLUQ, l'université à distance de l'Université du Québec à Monréal, et directrice du Centre d'expertise et de recherche sur l'apprentissage à vie (SAVIE). Mesdames Renaud et Sauvé sont également membres du projet Simulations and Advanced Gaming Environments for learning project / ApprentisSAGE par les jeux et simulations (SAGE).

\section{Correspondance}

renaud.lise@uqam.ca

lsauve@teluq.uqam.ca

\section{Contribution des auteures}

Lise Renaud: $50 \%$

Louise Sauvé: $50 \%$

Ce texte a été révisé par Ophélie Tremblay.

Texte reçu le: 7 avril 2009

Version finale reçue le: 6 juin 2010

Accepté le: 4 août 2010 